\title{
TRAIL protein expression in breast cancer cells correlates with nuclear grade
}

\author{
Adam Bilski', Grażyna Pasz-Walczak², Robert Kubiak², Piotr Sek¹, Justyna Chalubinska', \\ Wojciech Fendler ${ }^{3}$, Konrad Wronski ${ }^{4}$, Anna Piekarska ${ }^{5}$, Piotr Pluta ${ }^{1}$, Piotr Potemski ${ }^{6}$, \\ Arkadiusz Jeziorski ${ }^{1}$, Janusz Piekarski ${ }^{1}$
}

\begin{abstract}
1Department of Surgical Oncology, Chair of Oncology, Medical University of Lodz, Poland 2Department of Pathology, Chair of Oncology, Medical University of Lodz, Poland

${ }^{3}$ Department of Paediatrics, Haematology, Oncology and Diabetology, $1^{\text {st }}$ Chair of Paediatrics, Medical University of Lodz, Poland

${ }^{4}$ Department of Colorectal Surgery, $1^{\text {st }}$ Chair of Surgery, Medical University of Lodz, Poland ${ }^{5}$ Department and Chair of Infectious Diseases and Hepatology, Medical University of Lodz, Poland

${ }^{6}$ Department of Chemotherapy, Chair of Oncology, Medical University of Lodz, Poland
\end{abstract}

Submitted: 21 October 2009

Accepted: 2 January 2010

Arch Med Sci 2010; 6, 4: 545-551

DOI: 10.5114 /aoms.2010.14466

Copyright $\odot 2010$ Termedia \& Banach

\section{Abstract}

Introduction: TRAIL protein may serve as an escape mechanism for cancer cells from the immune response. The aim of the study was to assess whether the presence of TRAIL protein correlates with unfavourable prognostic factors in breast carcinoma.

Material and methods: The study group was composed of breast cancer patients treated surgically in the Department of Surgical Oncology, Medical University of Lodz, Poland, from January to December 2003. Inclusion criteria for the study were fulfilled by 117 women. The immunohistochemical study of TRAIL protein expression was performed in 118 breast carcinomas diagnosed in the study group. TRAIL protein expression was correlated with other variables: tumour size, lymph node status, grade, histological type of carcinoma, oestrogen and progesterone receptor status, HER2 expression, presence of lymphovascular invasion and age of the patient.

Results: Expression of TRAIL protein was present in $73 \%$ of breast carcinomas. The percentage of TRAIL-expressing breast carcinoma cells correlated with the nuclear grade $(\tau=0.26, p<0.05$; Tau Kendall test). The intensity of TRAIL expression (intensity of staining) in breast carcinoma cells correlated with the nuclear grade ( $\tau=0.15, p<0.05$; Tau Kendall test). TRAIL expression in breast carcinoma did not correlate with other studied variables.

Conclusions: Our analysis revealed that expression of TRAIL protein in breast carcinoma cells correlates with nuclear grade of carcinoma.

Key words: apoptosis, expression, immunohistochemistry.

\section{Introduction}

Apoptosis is a crucial mechanism of homeostasis in complex biological systems [1]. Three types of elements play an essential part in this process: activators, inhibitors, and effectors. The TRAIL protein, discovered in 1995 to be one of a family of tumour necrosis factors (TNF), acts as an activator of apoptosis [2]. The TRAIL ligand interacts with 5 receptors. Two of them,
Corresponding author: Janusz Piekarski MD, PhD Department of Surgical Oncology

Medical University of Lodz Paderewskiego 4

93-509 Lodz, Poland Phone: +48 426895441

Fax: +48426895422

E-mail:

januszpiekar@poczta.onet.pl 
death receptor-4 (DR4) and death receptor-5 (DR5), act agonistically; they activate apoptosis [3, 4]. The remaining 3 receptors, decoy receptor 1 (DcR1), decoy receptor 2 (DcR2) and osteoprotegerin, act antagonistically [5-7]. It was suggested that the TRAIL protein may have potent anticancer activity toward normal tissue without significant toxicity $[8,9]$, making it a potential anticancer drug. It was shown that TRAIL induces apoptosis in a variety of transformed cell lines. It has also been shown in an experimental study that the use of recombinant TRAIL protein is safe [10, 11].

The expression of TRAIL protein has been discovered in numerous normal cells and tissues in the human body. The expression of TRAIL has been found in cells of normal liver, kidneys, heart, colon, lungs, testes [12], and in immunological system cells [13]. In cancer cells, TRAIL protein expression may be higher or lower than in normal cells [14-17]. Nevertheless, the physiological role of the TRAIL protein in cancer cells is still not clear.

It has been suggested that expression of TRAIL protein may be a part of the escape mechanism for cancer cells from the immune response. TRAIL may induce apoptosis of activated lymphocytes [18], including tumour-infiltrating lymphocytes (TIL) [19]. Moreover, it has been demonstrated that the intensity of apoptosis correlates with the expression of another ligand from the TNF family, FasL, which is functionally similar to TRAIL [20-22]. In this aspect, the presence of TRAIL in cancer cells should be unfavourable for the patient as the decrease in number and decrease in cytotoxic activity of TIL deteriorate the prognosis [23].

On the other hand, expression of TRAIL protein in cancer cells may inhibit tumour growth by autocrine or paracrine induction of cancer cell apoptosis [24-26]. Moreover, induction of TRAIL expression may substantially increase paracrineled cancer cell apoptosis, without an increase of normal cell apoptosis [27]. The results of studies are not unanimous. Some of them show a lack of correlation between TRAIL expression and the intensity of cancer cell apoptosis [15, 28]. For all these reasons, it is very difficult to define the real role of TRAIL in cancer cells.

Assuming that the expression of TRAIL protein serves as an escape mechanism for cancer cells from the immune response, it can be expected that TRAIL should be an unfavourable prognostic factor. It would be interesting to assess whether the presence of TRAIL protein correlates with other unfavourable prognostic factors. It was therefore decided to search for such correlations in breast cancers.

\section{Material and methods}

The study group was composed of breast cancer patients treated surgically in the Department of
Surgical Oncology, Medical University of Lodz, Poland, from January to December 2003. The of each patient were examined for details of physical findings, type of surgery and results of pathological examination. Inclusion criteria for the present study were as follows: (1) axillary lymphadenectomy was performed during surgical treatment, as part of modified radical mastectomy or conservative surgery, (2) breast carcinoma was invasive, and (3) no neoadjuvant treatment was used. Moreover, all patients with metaplastic breast carcinoma were excluded. Complete clinical and pathological data, together with samples of archival breast cancer tissue, were obtained from 117 patients. As one of the breast carcinoma patients had bilateral breast carcinoma, the study was performed on 118 breast carcinoma cases. In order to examine the relationship between TRAIL expression and other clinical and pathological variables, an immunohistochemical study of TRAIL expression was performed. Immunohistochemical assessment of ER and PR expression, as well as assessment of HER2 expression in breast carcinomas, was carried out as part of a routine postoperative pathological workup. The results were the source for statistical calculations.

The study was approved by the ethical committee of the Medical University of Lodz (RNN/19/08/KE).

\section{Immunohistochemical stainings for TRAIL, ER, PR and HER2}

For immunohistochemical staining, 4- $\mu$ m-thick sections cut from formalin-fixed, paraffin-embedded tissue were used. After deparaffinization in xylene and hydration in graded ethanol solutions, the sections of breast carcinoma tissue for TRAIL, ER and PR staining were subjected to pre-treatment in a microwave oven in citrate buffer, in order to enhance antigen retrieval. The EnVision+ SystemHRP (DAB) (DakoCytomation) was used for the detection of immunostainings performed with primary antibodies against TRAIL antigen (Novocastra), ER and PR (Dako, Glostrup, Denmark). The immunostainings were performed according to the protocol described by the manufacturer. The dilutions of primary antibodies against TRAIL, against ER and against PR were 1 : 50. All incubations with the primary antibodies were conducted at $20-25^{\circ} \mathrm{C}$. Those for TRAIL lasted 30 min while those for ER and PR lasted 60 min. As a final step, counterstaining with haematoxylin was performed. Positive controls (antigen-positive tissue) for the immunostaining technique were used. The immunostaining for HER2 was performed with the use of HercepTest ${ }^{\circledR}$ (Dako, Glostrup, Denmark) strictly according to the protocol described by the manufacturer. All immunostainings 
were performed in the Department of Pathology, Chair of Oncology, Medical University of Lodz.

\section{Evaluation of TRAIL expression}

Two variants of evaluation of immunohistochemistry results were used. In the first, "simple" variant, TRAIL expression was classified as a binominal variable. The absence of TRAIL expression was defined as the absence of stained cells or presence of cytoplasmic staining in less than $10 \%$ of carcinoma cells. The presence of staining in at least $10 \%$ of carcinoma cells was considered as evidence of TRAIL expression.

In the second variant of evaluation, the percentage of stained cells and intensity of staining were classified separately. The percentage of stained cells was graded from 0 to 3 ( $0=$ less than $10 \%$ of cells stained, $1=10-39 \%, 2=40-69 \%, 3=$ at least $70 \%$ of cells stained). Intensity of staining was graded from 0 to 3 ( 0 = lack of staining or very weak intensity of staining; 1 = weak intensity of staining; 2 = moderate intensity of staining; 3 = strong intensity of staining).

\section{Evaluation of ER and PR expression}

The scoring of ER and PR nuclear reactivity was done using the method described by McCarty et al. [29]. Both the percentage of stained cells and the intensity of reaction were taken into account. Histoscore (HS) was calculated for each carcinoma using the mathematical formula:

where:

$$
\mathrm{HS}=x(+)+2 x(++)+3 x(+++)
$$

$x(+)$ was the percentage of cells with weak intensity of staining,

$x(++)$ was the percentage of cells with moderate intensity of staining,

$x(+++)$ was the percentage of cells with strong intensity of staining.

The expression of oestrogen receptor and expression of progesterone receptor were graded in relation to histo-score value: histo-score 0-100 $=(0)$ absence of expression, histo-score 101-200 $=(1+)$ weak expression, histo-score 201-300 $=(2+)$ moderate expression, histo-score 301-400 $=(3+)$ strong expression.

\section{Evaluation of HER2 staining}

The scoring system recommended by the manufacturer of the HercepTest (Dako, Glostrup, Denmark) was used for the evaluation of HER2 staining. HER2 was scored 0 when no staining was observed or membrane staining was observed in less than $10 \%$ of the carcinoma cells. It was scored $1+$ when faint/barely perceptible membrane staining was detected in more than $10 \%$ of carcinoma cells, but cells exhibited incomplete membrane staining.
It was scored 2+ when weak to moderate complete membrane staining was observed in more than $10 \%$ of carcinoma cells. It was scored $3+$ when strong complete membrane staining was observed in more than $10 \%$ of carcinoma cells.

\section{Statistical analysis}

Statistical analysis was performed using STATISTICA 8.0 statistical package (StatSoft, Tulsa, USA). Two-tailed, exact Fisher's test or Yates corrected $\chi^{2}$ test was used depending on the number of observations. The normality of the distribution of continuous variables was verified using Shapiro-Wilk $W$ test. In cases of non-normally distributed values, logarithmic transformation was attempted. Nonparametric tests were used if the distribution remained non-normal. Correlation computations were performed using the Spearman $R$ and Tau Kendall tests. In all cases, the threshold of statistical significance was the probability of a type I error lower than 0.05 .

\section{Results \\ Patients}

In the study group, 118 breast carcinomas were diagnosed in 117 women. The median age of patients at the time of diagnosis of breast carcinoma was 56 years (range, 26-81 years). Breast carcinomas were located in the left breast in 57 cases $(48.3 \%)$ and in the right breast in 61 cases (51.7\%).

\section{Breast carcinomas}

Invasive ductal carcinoma was the dominant type of cancer. It was diagnosed in 90 cases $(90 / 118$, $76.3 \%)$. In 21 cases invasive lobular carcinoma was diagnosed (21/118, 17.8\%). Among the remaining 7 carcinomas (7/118, 5.9\%), histological types were as follows: invasive ductal-lobular (3 cases), medullary (2 cases), mucinous (1 case), and alveolar (1 case) (WHO 2004 histological classification). The nuclear grade was classified as G3 in 54 carcinomas, as $G 2$ in 50 carcinomas and as G1 in 14 carcinomas. The median size of the primary tumours was $2.25 \mathrm{~cm}$ (range $0.5-8 \mathrm{~cm}$, mean $2.4 \mathrm{~cm}$, standard deviation $1.32 \mathrm{~cm}$ ). Metastases in axillary lymph nodes were found in 59 cases (59/118, 50.0\%). In the remaining 59 cases, no metastases were found (59/118, 50.0\%). In 33 cases, metastases in lymph nodes infiltrated the lymph node capsule (33/118, $28.0 \%)$. Lymphovascular invasion was found in 17 cases $(17 / 118,14.4 \%)$. In the whole group of 118 primary carcinomas, 33 were classified as stage I carcinomas (33/118, 28.0\%), 41 as IIA carcinomas (41/118, 34.8\%), 43 as IIB carcinomas (43/118, $36.4 \%)$ and one as IIIA carcinoma (1/118, 0.8\%). 


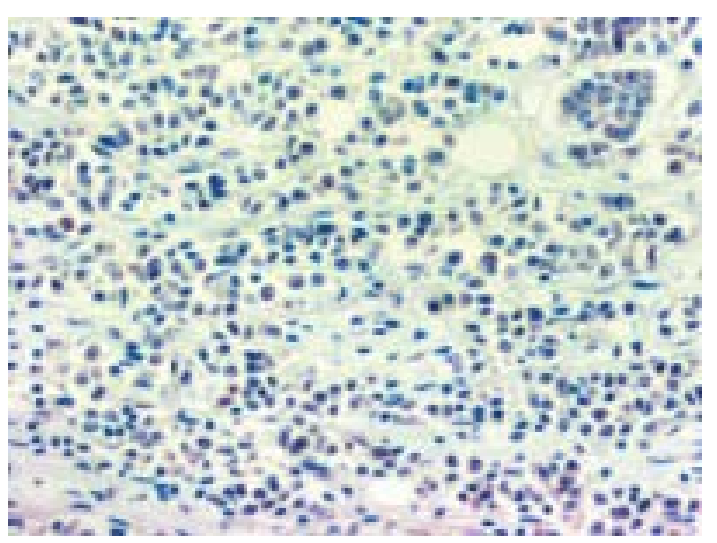

Figure 1. Absence of cytoplasmic TRAIL expression in breast carcinoma cells. Magnification 200x

\section{Results of immunohistochemical stainings for TRAIL, ER, PR and HER2}

In 32 primary breast carcinomas, TRAIL expression was absent $(32 / 118,27.1 \%)$ (Figure 1). In the remaining 86 of 118 primary breast carcinomas, the expression of TRAIL was present $(86 / 118,72.9 \%)$ (Figure 2). The results of immunohistochemical staining for TRAIL are presented in detail in Table I. The results of immunohistochemical stainings for ER, PR and HER2 are presented in detail in Table II.

\section{Correlations of TRAIL expression}

The percentage of TRAIL-expressing breast carcinoma cells correlated with the nuclear grade ( $\tau=0.26, p<0.05$; Tau Kendall test). Also the intensity of TRAIL expression (intensity of staining) in breast carcinoma cells correlated with the nuclear grade ( $\tau=0.15, p<0.05$; Tau Kendall test).

The percentage of TRAIL-expressing breast carcinoma cells did not correlate with the diameter of the primary tumour $(R=0.04, p>0.05$; Spearman $R$ test), oestrogen receptor expression $(\tau=-0.04, p>0.05$; Tau Kendall test), progesterone receptor expression $(\tau=-0.08, p>0.05$; Tau Kendall

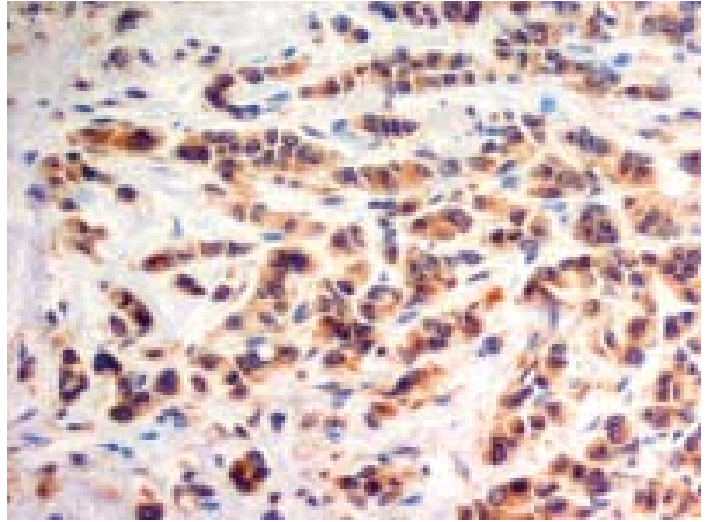

Figure 2. Presence of cytoplasmic TRAIL expression in majority of breast carcinoma cells. Magnification 200x

test), HER2 score ( $\tau=0.1, p>0.05$; Tau Kendall test), or age of breast carcinoma patients $(R=-0.07$, $p>0.05$; Spearman $R$ test).

The intensity of TRAIL expression (intensity of staining) in breast carcinoma cells did not correlate with the diameter of the primary tumour $(R=0.03$, $p>0.05$; Spearman $R$ test), oestrogen receptor expression ( $\tau=-0.06, p>0.05$; Tau Kendall test), progesterone receptor expression $(\tau=-0.02$, $p>0.05$; Tau Kendall test), HER2 score $(\tau=-0.01$, $p>0.05$; Tau Kendall test), or age of breast carcinoma patients $(R=0.06, p>0.05$; Spearman $R$ test).

The presence of TRAIL expression in breast carcinoma cells was not statistically associated with the histological type of breast carcinoma $(p=0.73)$, with the presence of lymphovascular invasion $(p=0.95)$, with the presence of metastases in axillary lymph nodes $(p=0.53)$ or with infiltration of the lymph node capsule by lymph node metastases ( $p=0.1$ ) (all tests: two-tailed exact Fisher's test or Yates corrected $\chi^{2}$ test).

Similar statistical analysis was also performed in the subgroup of all 90 ductal invasive breast carcinomas. The results were similar to those of the

Table I. Results of immunohistochemical study of TRAIL expression in 118 primary breast carcinomas

\begin{tabular}{|c|c|c|}
\hline Expression of TRAIL in breast carcinoma & Number of cases & Percentage of cases \\
\hline \multicolumn{3}{|c|}{ Percentage of stained cells } \\
\hline Less than $10 \%$ of cells (grade 0 ) & $32 / 118$ & 27.1 \\
\hline $10-39 \%$ of cells (grade $1+$ ) & $13 / 118$ & 11.0 \\
\hline $40-69 \%$ of cells (grade $2+$ ) & $26 / 118$ & 22.0 \\
\hline At least $70 \%$ of cells (grade $3+$ ) & $47 / 118$ & 39.8 \\
\hline \multicolumn{3}{|c|}{ Intensity of staining } \\
\hline Absence of staining (grade 0 ) & $32 / 118$ & 27.1 \\
\hline Weak intensity of staining (grade $1+$ ) & $24 / 118$ & 20.3 \\
\hline Moderate intensity of staining (grade $2+$ ) & $43 / 118$ & 36.4 \\
\hline Strong intensity of staining (grade $3+$ ) & $19 / 118$ & 16.1 \\
\hline
\end{tabular}


Table II. Results of immunohistochemical stainings for oestrogen, progesterone and HER2 receptors in 118 primary breast carcinomas

\begin{tabular}{|c|c|c|}
\hline Studied antigens & Number of cases & Percentage of cases \\
\hline \multicolumn{3}{|c|}{ Expression of oestrogen receptor } \\
\hline Grade 0 & 51 & 43.2 \\
\hline Grade 1+ & 18 & 15.3 \\
\hline Grade 2+ & 22 & 18.6 \\
\hline Grade 3+ & 19 & 16.1 \\
\hline \multicolumn{3}{|c|}{ Expression of progesterone receptor } \\
\hline Grade 0 & 54 & 45.8 \\
\hline Grade 1+ & 11 & 9.3 \\
\hline Grade 2+ & 24 & 20.3 \\
\hline Grade 3+ & 29 & 24.6 \\
\hline \multicolumn{3}{|c|}{ HER2 receptor score } \\
\hline Score 0 & 48 & 40.7 \\
\hline Score 1+ & 23 & 19.5 \\
\hline Score 2+ & 27 & 22.9 \\
\hline Score $3+$ & 20 & 16.9 \\
\hline
\end{tabular}

whole breast carcinoma group. The percentage of TRAIL-expressing cells of ductal invasive breast carcinomas correlated with the nuclear grade ( $\tau=0.32, p<0.05$; Tau Kendall test). Also the intensity of TRAIL expression (intensity of staining) in cells of ductal invasive breast carcinomas correlated with the nuclear grade $(\tau=0.19, p<0.05$; Tau Kendall test). The results of calculations performed for remaining clinical and pathological variables were statistically insignificant $(p>0.05)$. The statistical analyses of subgroups of other histological types of breast carcinomas were abandoned. The number of breast carcinomas in each of the non-ductal invasive carcinoma subgroups was too small to achieve reliable statistical data.

\section{Discussion}

Our analysis revealed that the expression of TRAIL protein in breast carcinoma cells correlated with nuclear grade of carcinoma. We found such a correlation for both the percentage of TRAILexpressing cells and for the intensity of TRAIL staining. To the best of our knowledge, this study is the first presentation of the correlation of TRAIL expression with nuclear grade in breast carcinoma in medical literature. The few authors who have studied TRAIL expression in breast carcinoma did not find any correlation with tumour grade [30, 31].

In our study, TRAIL expression did not correlate nor was associated with tumour size, axillary lymph node status, histological type of carcinoma, or expression of oestrogen receptor and progesterone receptor. In this aspect, our observations are similar to observations made by other authors. Lack of correlation between TRAIL expression and tumour size, axillary lymph node status and histological type of carcinoma was reported by Cross et al. [30]. Lack of correlation between TRAIL and the expression of oestrogen receptor and progesterone receptor was reported by Cross et al. [30], by Sanlioglu et al. [31] and by Van Poznak et al. [32]. In our study we did not find an association between TRAIL expression and HER2 score. In this aspect our results are different from the results presented by Sanlioglu et al. [31], who reported an association between TRAIL protein and HER2 overexpression in breast carcinoma.

In our study we found that TRAIL-expressing breast carcinomas constitute $73 \%$ of all studied cases. This percentage is higher than the percentages reported by Herrnring et al. [14] and Van Poznak et al. [32], who found expression of TRAIL protein in $52-58 \%$ of breast carcinomas. It is possible that the difference between our results and the results reported by Herrning et al. derives from differences in methodology [14]. Herrnring et al. did not use the microwave pre-treatment employed in this study. However, Van Poznak et al. [32] used a very similar methodology of immunohistochemical staining to ours; moreover, our method of evaluation of TRAIL protein expression was also very similar to the method used by Van Poznak et al. [32]. Nevertheless, the difference between the results remains substantial (Van Poznak et al., 58\% vs. our study, 73\%). The size 
of our study group (118 breast carcinomas) is not small. In fact it is larger than the sizes of groups analysed by Van Poznak et al. [32] (40 carcinomas), by Sanlioglu et al. [31] (90 carcinomas) and by Herrnring et al. [14] (40 carcinomas). All these authors used classic immunohistochemistry in their studies, as we did. Therefore, it is tempting to speculate that we were able to identify a correlation between grade of breast carcinoma and TRAIL expression due to the larger number of studied cases. However, in one very large immunohistochemical study performed with the tissue microarray technique, Cross et al. analysed 395 breast carcinomas and did not find an association between grade of breast carcinoma and TRAIL expression [30]. For this reason, we are sure that the cause of the differences between the studies lies elsewhere. It is also possible that different methods of grading of breast carcinomas could be responsible for the differences in results achieved in the discussed studies: in our study, the presence of correlations was assessed with the nuclear grade of cancer; other authors, such as Sanlioglu et al. [31], used histopathological grading (Elston-Ellis method [33]).

In conclusion, our analysis revealed that the expression of TRAIL protein in breast carcinoma correlates with a potent unfavourable prognostic factor - cancer grade. A similar observation was presented for lung cancer [34]. In melanoma, TRAIL expression was correlated with high mitotic index [35]. All these observations indicate generally that TRAIL protein may be typical for high grade carcinomas. However, it is still very difficult to define the role of TRAIL protein in carcinoma cells. There are two main possibilities. Firstly, TRAIL acts as an unfavourable factor suppressing cytotoxic activity of tumour-infiltrating lymphocytes; it helps the escape of cancer cells from the immune response $[18,19]$. Secondly, TRAIL acts as a favourable factor, serving as a mechanism of cancer self-regulation; it inhibits tumour growth by autocrine or paracrine induction of cancer cell apoptosis [24-26].

\section{Acknowledgments}

This study was supported by the Medical University of Lodz, grant No. 501-11-743.

\section{References}

1. Steller $\mathrm{H}$. Mechanisms and genes of cellular suicide. Science 1995; 267: 1445-9.

2. Wiley SR, Schooley K, Smolak PJ, et al. Identification and characterization of a new member of the TNF family that induces apoptosis. Immunity 1995; 3: 673-82.

3. Pan G, O'Rourke K, Chinnaiyan AM, et al. The receptor for the cytotoxic ligand TRAIL. Science 1997; 276: 111-3.

4. Sheridan JP, Marsters SA, Pitti RM, et al. Control of TRAILinduced apoptosis by a family of signaling and decoy receptors. Science 1997; 277: 818-21.
5. Degli-Esposti MA, Dougall WC, Smolak PJ, Waugh JY, Smith CA, Goodwin RG. The novel receptor TRAIL-R4 induces NFkappaB and protects against TRAIL-mediated apoptosis, yet retains an incomplete death domain. Immunity 1997; 7: 813-20.

6. Degli-Esposti MA, Smolak PJ, Walczak H, et al. Cloning and characterization of TRAIL-R3, a novel member of the emerging TRAIL receptor family. J Exp Med 1997; 186: 1165-70.

7. Emery JG, McDonnell P, Burke MB, et al. Osteoprotegerin is a receptor for the cytotoxic ligand TRAIL. J Biol Chem 1998; 273: 14363-7.

8. Ashkenazi A, Pai RC, Fong S, et al. Safety and antitumor activity of recombinant soluble Apo2 ligand. J Clin Invest 1999; 104: 155-62.

9. Walczak H, Miller RE, Ariail K, et al. Tumoricidal activity of tumor necrosis factor-related apoptosis-inducing ligand in vivo. Nat Med 1999; 5: 157-63.

10. Kelley SK, Harris LA, Xie D, et al. Preclinical studies to predict the disposition of Apo2L/tumor necrosis factorrelated apoptosis-inducing ligand in humans: characterization of in vivo efficacy, pharmacokinetics, and safety. J Pharmacol Exp Ther 2001; 299: 31-8.

11. Fang F, Wang AP, Yang SF. Antitumor activity of a novel recombinant mutant human tumor necrosis factor-related apoptosis-inducing ligand. Acta Pharmacol Sin 2005; 26: 1373-81.

12. Spierings DC, de Vries EG, Vellenga E, et al. Tissue distribution of the death ligand TRAIL and its receptors. J Histochem Cytochem 2004; 52: 821-31.

13. Kamohara H, Matsuyama W, Shimozato O, et al. Regulation of tumour necrosis factor-related apoptosisinducing ligand (TRAIL) and TRAIL receptor expression in human neutrophils. Immunology 2004; 111: 186-94.

14. Herrnring C, Reimer T, Jeschke U, et al. Expression of the apoptosis-inducing ligands FasL and TRAIL in malignant and benign human breast tumors. Histochem Cell Biol 2000; 113: 189-94.

15. Koornstra JJ, Kleibeuker JH, van Geelen CM, et al. Expression of TRAIL (TNF-related apoptosis-inducing ligand) and its receptors in normal colonic mucosa, adenomas, and carcinomas. J Pathol 2003; 200: 327-35.

16. Sanlioglu AD, Koksal IT, Ciftcioglu A, Baykara M, Luleci G, Sanlioglu S. Differential expression of TRAIL and its receptors in benign and malignant prostate tissues. J Urol 2007; 177: 359-64.

17. Vigneswaran N, Baucum DC, Wu J, et al. Repression of tumor necrosis factor-related apoptosis-inducing ligand (TRAIL) but not its receptors during oral cancer progression. BMC Cancer 2007; 7: 108.

18. Inoue H, Shiraki K, Yamanaka T, et al. Functional expression of tumor necrosis factor-related apoptosisinducing ligand in human colonic adenocarcinoma cells. Lab Invest 2002; 82: 1111-9.

19. Koyama S, Koike N, Adachi S. Expression of TNF-related apoptosis-inducing ligand (TRAIL) and its receptors in gastric carcinoma and tumor-infiltrating lymphocytes: a possible mechanism of immune evasion of the tumor. J Cancer Res Clin Oncol 2002; 128: 73-9.

20. Céfai D, Favre L, Wattendorf E, Marti A, Jaggi R, Gimmi CD. Role of Fas ligand expression in promoting escape from immune rejection in a spontaneous tumor model. Int J Cancer 2001; 91: 529-37.

21. Cheng B. Association between up-regulation of Fas ligand expression and apoptosis of tumor-infiltrating lymphocytes in human breast cancer. J Huazhong Univ Sci Technolog Med Sci 2006; 26: 573-5. 
22. Ioachim HL, Decuseara R, Giancotti F, Dorsett BH. FAS and FAS-L expression by tumor cells and lymphocytes in breast carcinomas and their lymph node metastases. Pathol Res Pract 2005; 200: 743-51.

23. O'Connell J, Bennett MW, O'Sullivan GC, O'Callaghan J, Collins JK, Shanahan F. Expression of Fas (CD95/APO-1) ligand by human breast cancers: significance for tumor immune privilege. Clin Diagn Lab Immunol 1999; 6: 457-63.

24. Griffith TS, Anderson RD, Davidson BL, Williams RD, Ratliff TL. Adenoviral-mediated transfer of the TNF-related apoptosis-inducing ligand/Apo-2 ligand gene induces tumor cell apoptosis. J Immunol 2000; 165: 2886-94.

25. Abadie A, Wietzerbin J. Involvement of TNF-related apoptosis-inducing ligand (TRAIL) induction in interferon gamma-mediated apoptosis in Ewing tumor cells. Ann N Y Acad Sci 2003; 1010: 117-20.

26. Papageorgiou A, Lashinger L, Millikan R, et al. Role of tumor necrosis factor-related apoptosis-inducing ligand in interferon-induced apoptosis in human bladder cancer cells. Cancer Res 2004; 64: 8973-9.

27. Clarke N, Jimenez-Lara AM, Voltz E, Gronemeyer H. Tumor suppressor IRF-1 mediates retinoid and interferon anticancer signaling to death ligand TRAIL. EMBO J 2004; 23: $3051-60$

28. Koornstra JJ, Jalving M, Rijcken FE, et al. Expression of tumour necrosis factor-related apoptosis-inducing ligand death receptors in sporadic and hereditary colorectal tumours: potential targets for apoptosis induction. Eur J Cancer 2005; 41: 1195-202.

29. McCarty KS Jr, Miller LS, Cox EB, Konrath J, McCarty KS Estrogen receptor analyses. Correlation of biochemical and immunohistochemical methods using monoclonal antireceptor antibodies. Arch Pathol Lab Med 1985; 109: 716-21.

30. Cross SS, Harrison RF, Balasubramanian SP, et al. Expression of receptor activator of nuclear factor kappabeta ligand (RANKL) and tumour necrosis factor related, apoptosis inducing ligand (TRAIL) in breast cancer, and their relations with osteoprotegerin, oestrogen receptor, and clinicopathological variables. J Clin Pathol 2006; 59: 716-20.

31. Sanlioglu AD, Korcum AF, Pestereli E, et al. TRAIL death receptor-4 expression positively correlates with the tumor grade in breast cancer patients with invasive ductal carcinoma. Int J Radiat Oncol Biol Phys 2007; 69: 716-23.

32. Van Poznak C, Cross SS, Saggese M, et al. Expression of osteoprotegerin (OPG), TNF related apoptosis inducing ligand (TRAIL), and receptor activator of nuclear factor kappaB ligand (RANKL) in human breast tumours. J Clin Pathol 2006; 59: 56-63.

33. Elston CW, Ellis IO. Assessment of histological grade. In: Elston CW, Ellis IO, editors. The breast, Churchill Livingstone, Edinburgh 1998.

34. Spierings DC, de Vries EG, Timens W, Groen HJ, Boezen HM, de Jong S. Expression of TRAIL and TRAIL death receptors in stage III non-small cell lung cancer tumors. Clin Cancer Res 2003; 9: 3397-405.

35. Bron LP, Scolyer RA, Thompson JF, Hersey P. Histological expression of tumour necrosis factor-related apoptosisinducing ligand (TRAIL) in human primary melanoma. Pathology 2004; 36: 561-5. 